\title{
METHODOLOGICAL FEATURES OF DETERMINING THE LEVEL OF COMPETITIVENESS IN THE AGRI-FOOD SECTOR OF GEORGIA
}

\author{
ETER KHARAISHVILI \\ Doctor of Economic Sciences, professor, \\ Ivane Javakhishvili Tbilisi State University, \\ Academician of the Academy of Economic \\ Sciences of Georgia, Georgia \\ eter.kharaishvili@tsu.ge
}

Abstract. The paper evaluates the importance of scientifically calculated indicators of competition and level of competitiveness for the effective functioning of food markets, especially for Georgia. The role of these indicators for producers and consumers is also substantiated.

The methodological issues of measuring the level of competition in food markets are evaluated in the paper. The opinions of various researchers on this issue are analyzed. It is concluded that in Georgia, where the share of small enterprises is large and the import rate is high, in order to estimate the market share of small firms along with the concentration index, it is advisable to calculate the Herfindahl-Hirschman indices, as well as the entropy index and to determine the dispersion of market shares.

To provide a comprehensive assessment of the level of competition and to provide complete information to stakeholders, it is advisable to use other features as well. It is argued that determining the level of competition requires, in addition to the indices, the analysis of various competitive factors. The article evaluates five key competitive factors based on wine market analysis: local market position; Local market growth potential; Economies of scale (cost / benefit analysis); Adapting to industry change; Potential for attracting foreign investors. The obtained results are compared with the data of the research conducted by foreign scientists according to the groups of traditional and new wine producers. Comparative analysis reveals methodological problems in determining the level of competition for the agro-food sector of Georgia.

In order to successfully enter the food markets, it is advisable to calculate the level of competitiveness of the product. The level of product competitiveness in the current period is assessed by the "ex-post" method using the following indices: Revealed Competitiveness Advantage (RCA); Relative Advantages of Exports (RXA); By Relative Import Penetration (RMP); By Relative Trade Advantage (RTA). It is concluded that for Georgia, as a small country, to determine the level of competitiveness of agri-food products from two indices (RCA and RXA indices) it is recommended to use only the RCA index.

The article identifies modern methodological approaches to determining the level of competition and competitiveness, draws conclusions based on their evaluation and offers recommendations for overcoming different problems.

KEYWORDS: COMPETITION, AGRI-FOOD SECTOR, COMPETITIVENESS, COMPETITIVE FACTORS, COMPETITIVENESS INDICES.

For citation: Kharaishvili, E., (2020). Methodological Features of Determining the Level of Competition and Competitiveness in the Agri-food Sector of Georgia. Globalization and Business, 10. 42-48. (In Georgian) https://doi.org/10.35945/gb.2020.10.004 


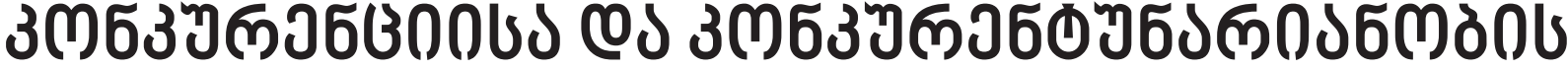

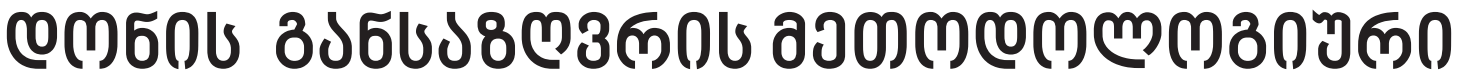

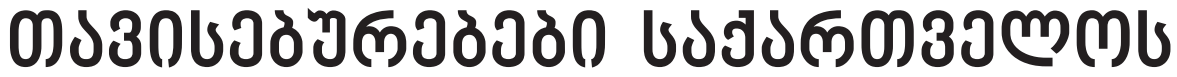

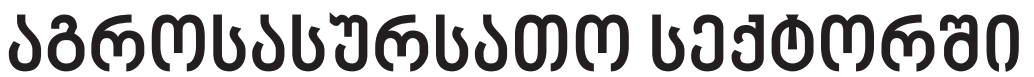

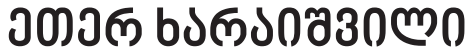

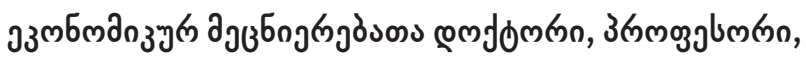

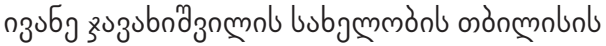

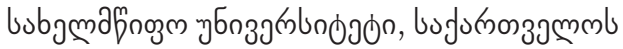

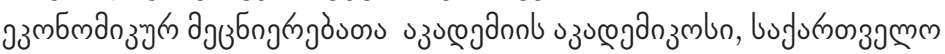
eter.kharaishvili@tsu.ge

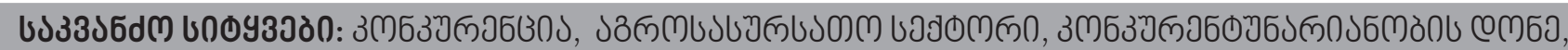

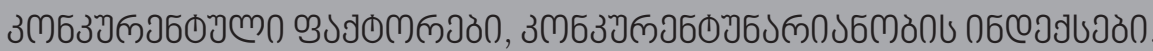

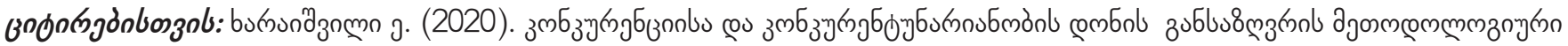

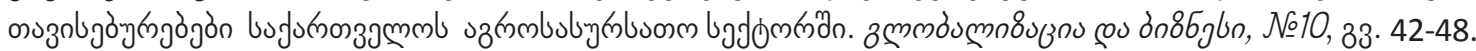

https://doi.org/10.35945/gb.2020.10.004

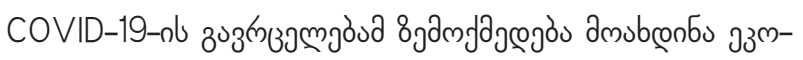

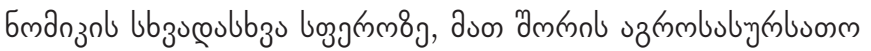

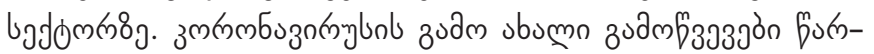

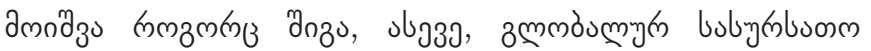
os8

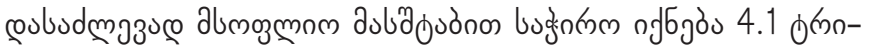

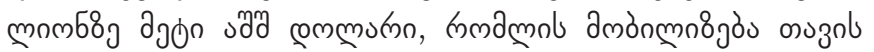

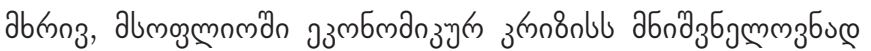

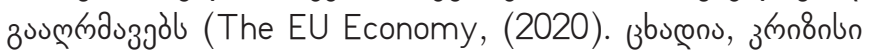

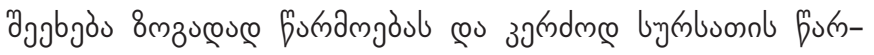

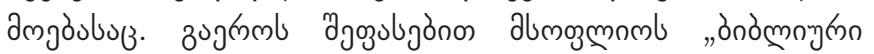

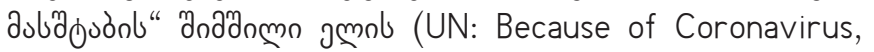

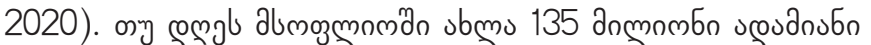

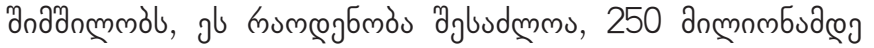

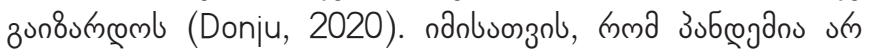

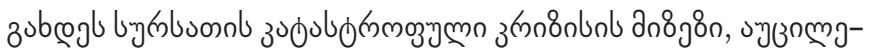

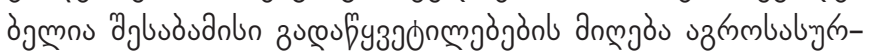

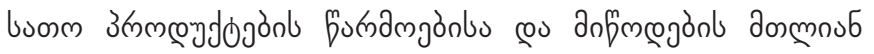

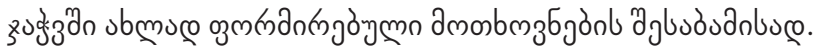

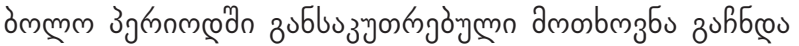

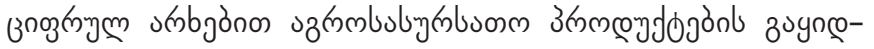

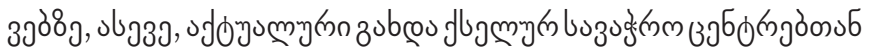

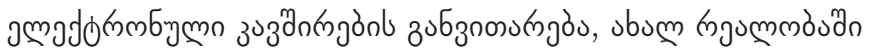

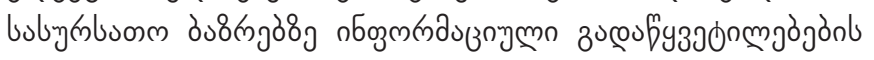

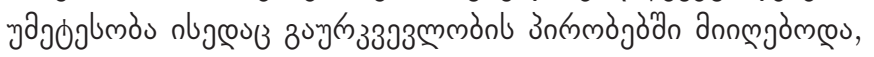

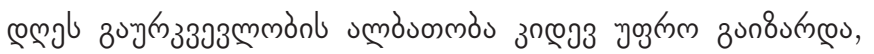

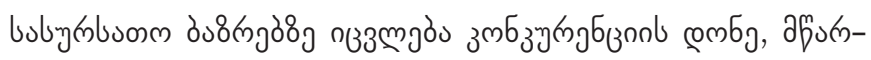

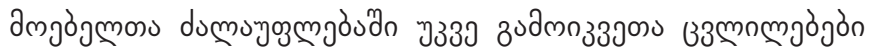

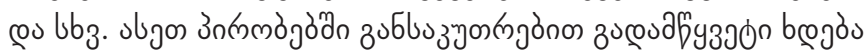

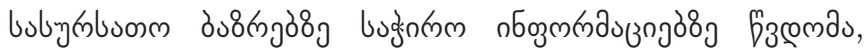

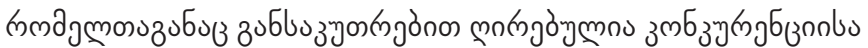

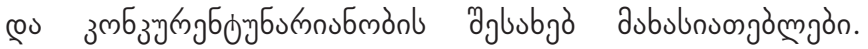

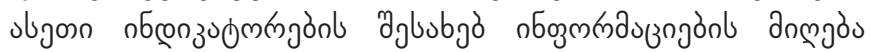

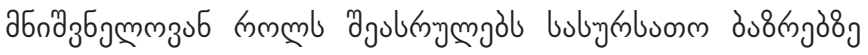

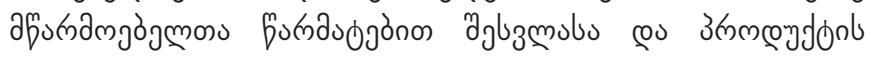

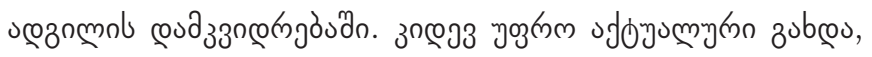

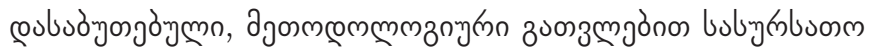

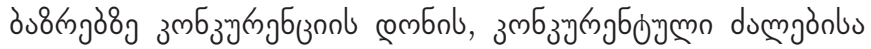

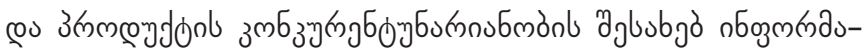

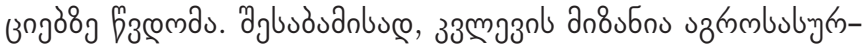
bsomm bajom

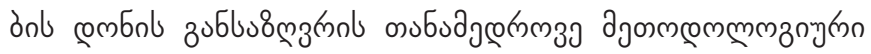

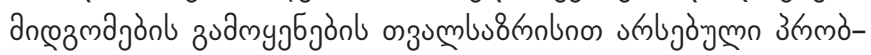

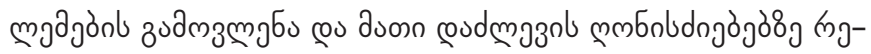

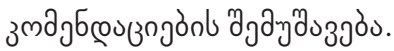

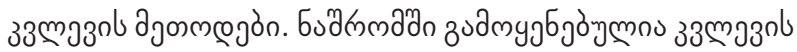

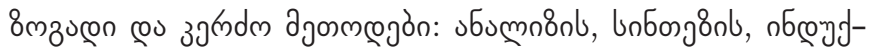

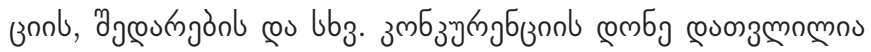

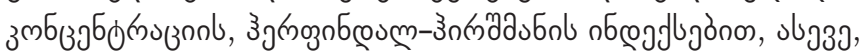

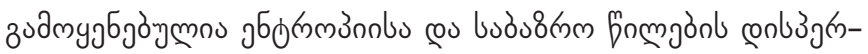

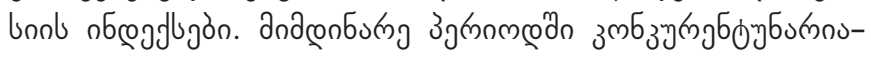

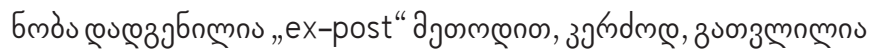




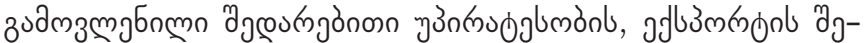

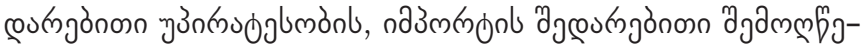

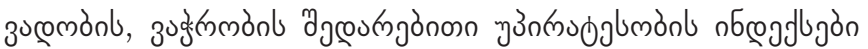
coubs.

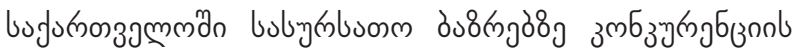

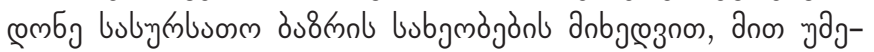

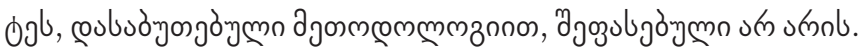

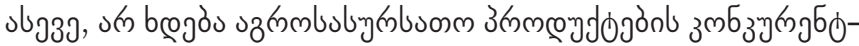

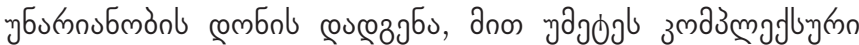

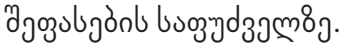

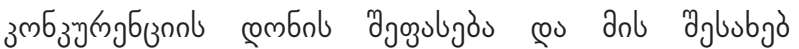

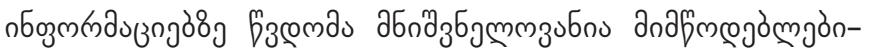

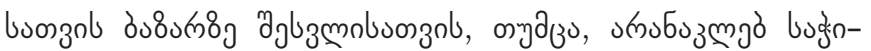

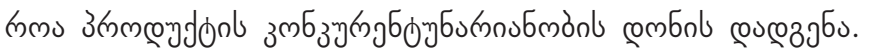

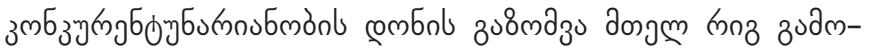

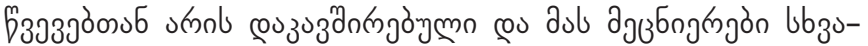

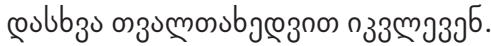

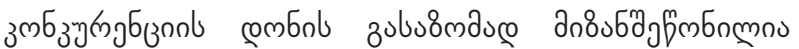

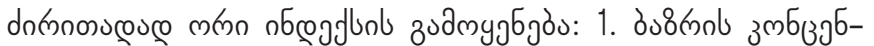

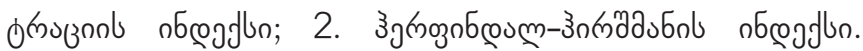

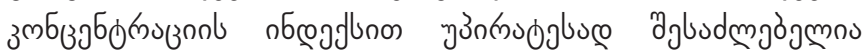

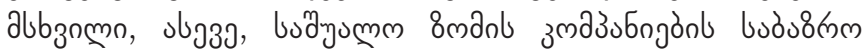
fremol zublu8 zumn zodm

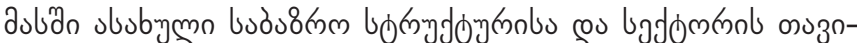

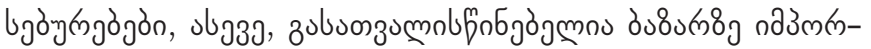

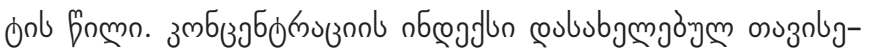

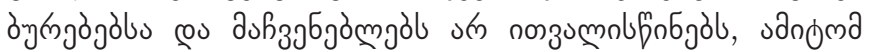

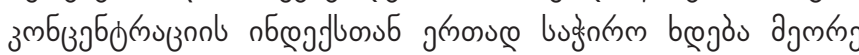

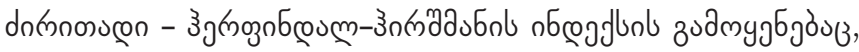

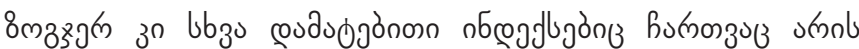

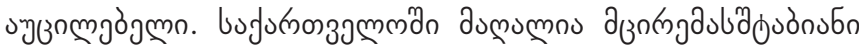

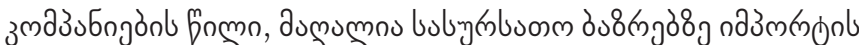

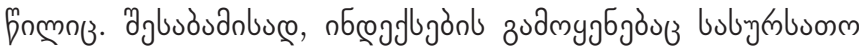

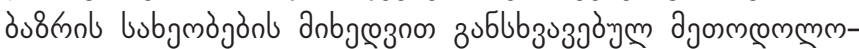

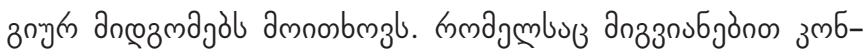

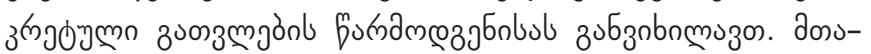

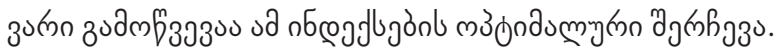

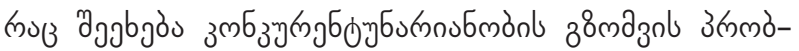

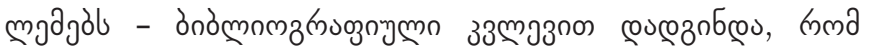
a

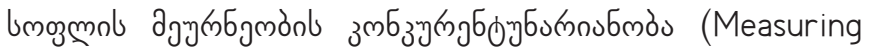

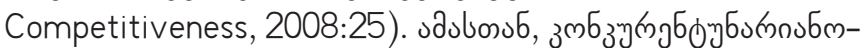
on buf

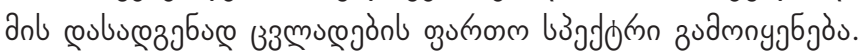

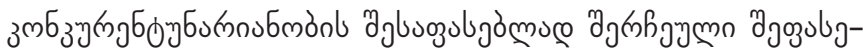

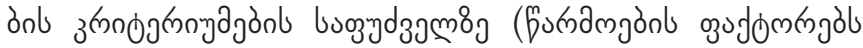

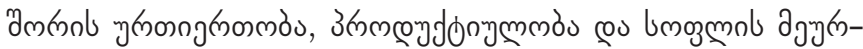

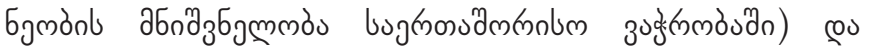

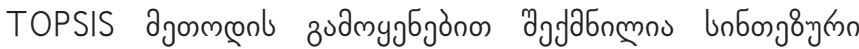

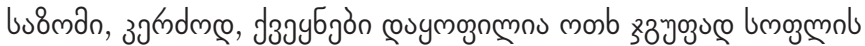

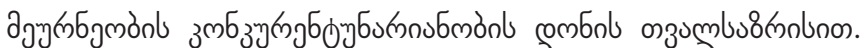

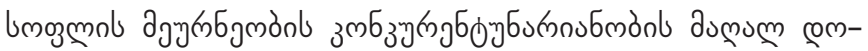

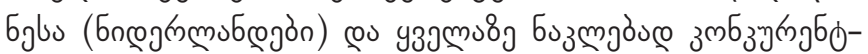

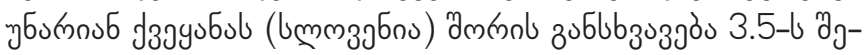

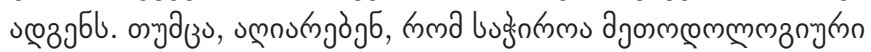

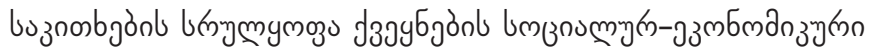

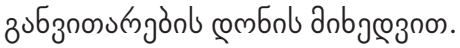

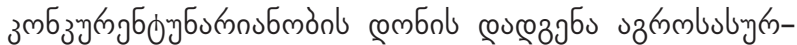
buom badjm

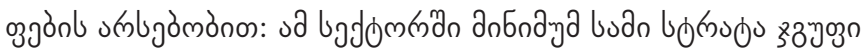
zutonbongojo:

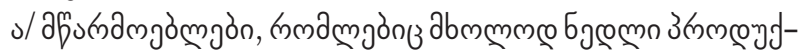

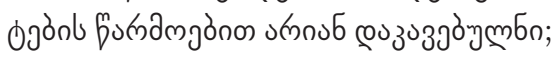

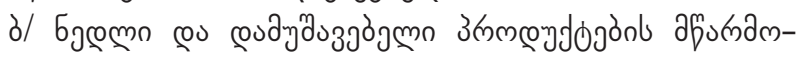
jòmgòn;

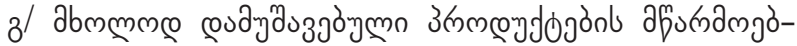
mjòn.

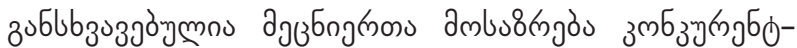

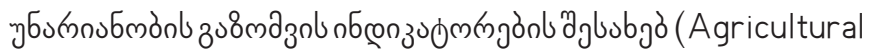

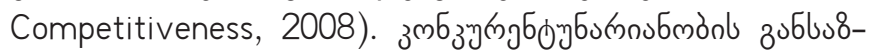

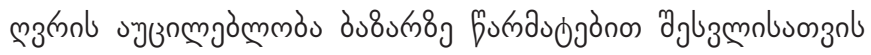

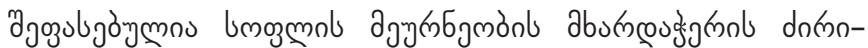

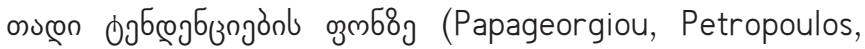

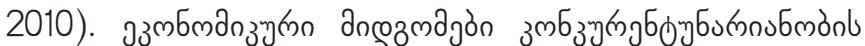

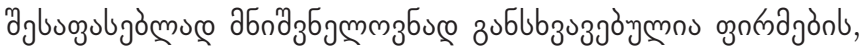

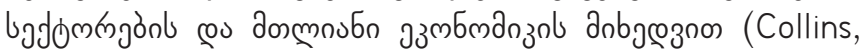
2004).

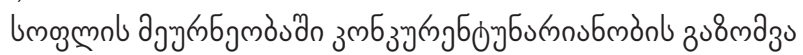

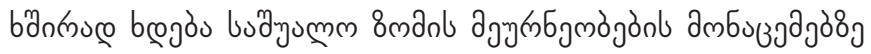
cougnnobmónon (Maslova, Zaruk, Fuchs, Avdeev, 2018).

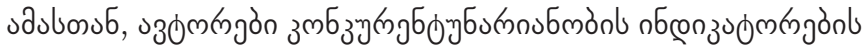

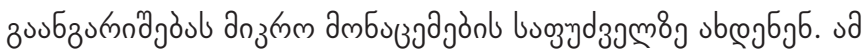

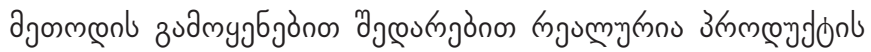

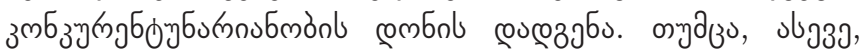

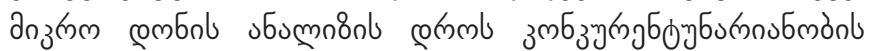

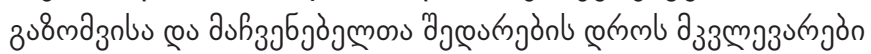

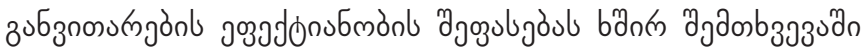

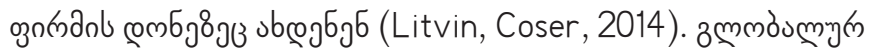

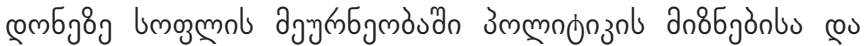

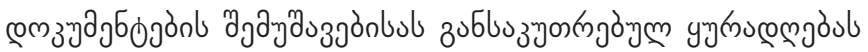

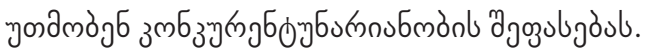

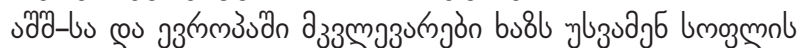

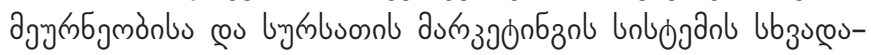
ubzu jou38 3 змб oub, зmб

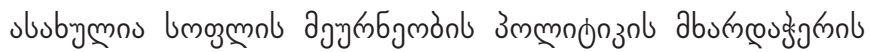

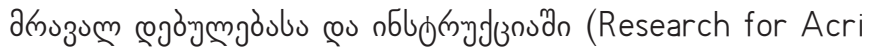
Committee, 2018).

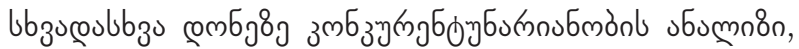




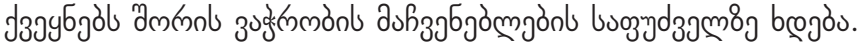

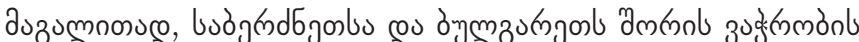

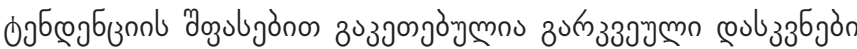

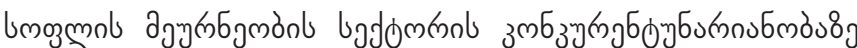
(Nivievski,Cramon,2009; Nowak, Kaminska, 2016).

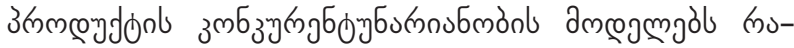

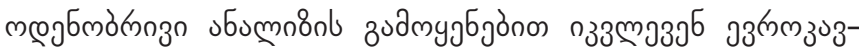

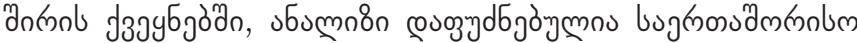

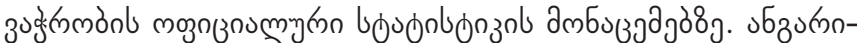

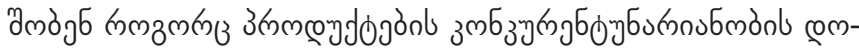

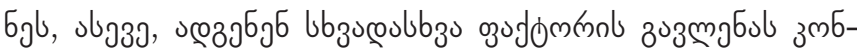

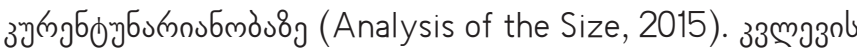

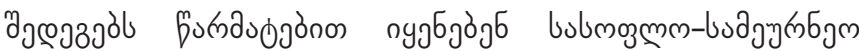

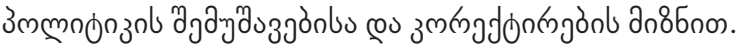

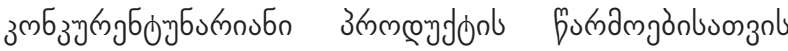

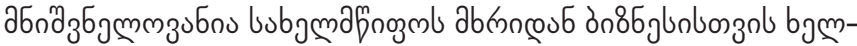

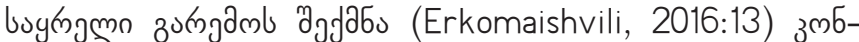

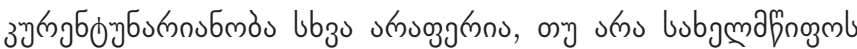

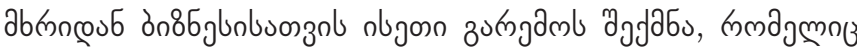

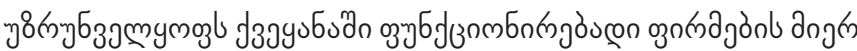

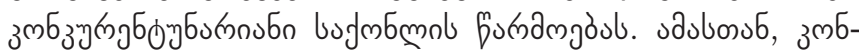

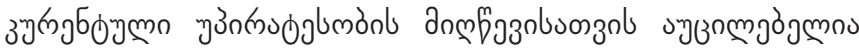

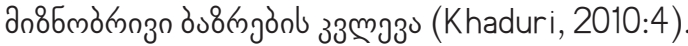

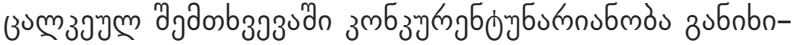

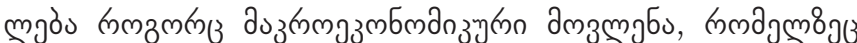

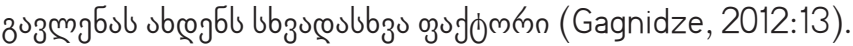

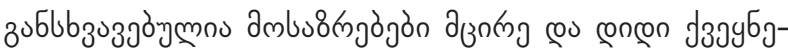

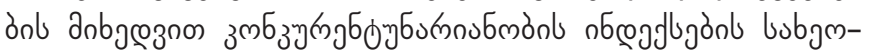

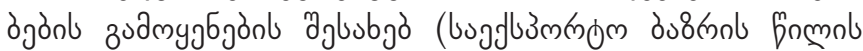

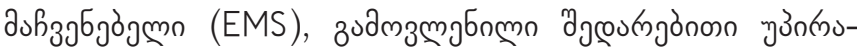

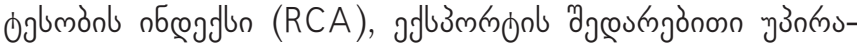

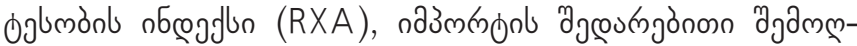

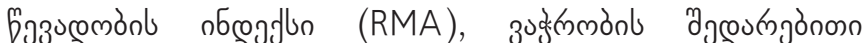

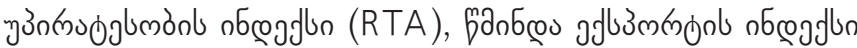

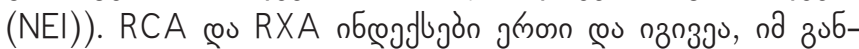

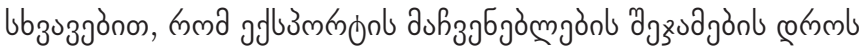

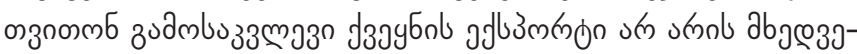

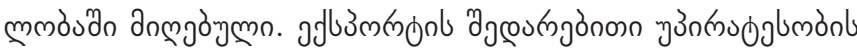

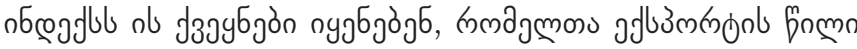

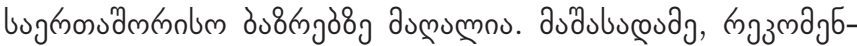

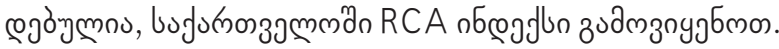

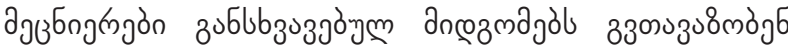
ง

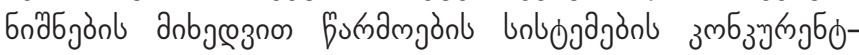

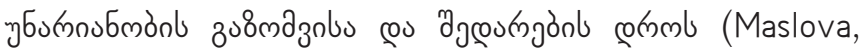

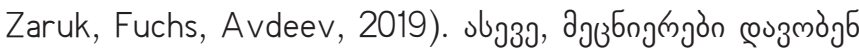

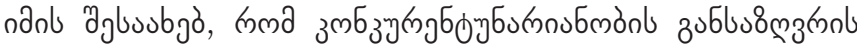

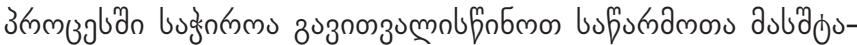

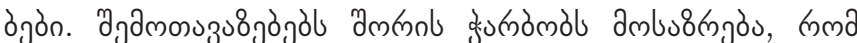

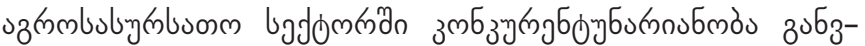

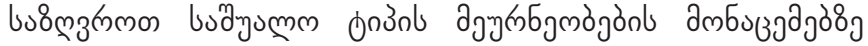

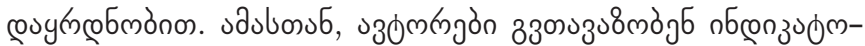

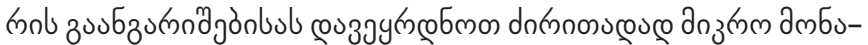

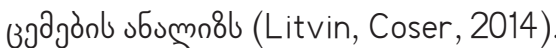

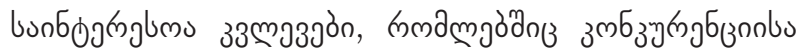

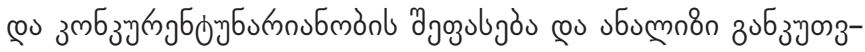

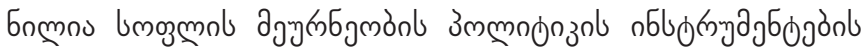

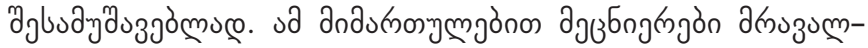

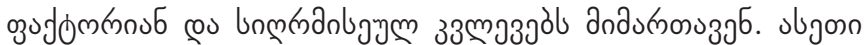

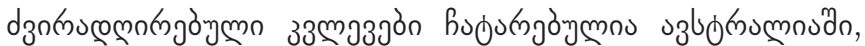

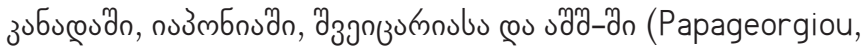

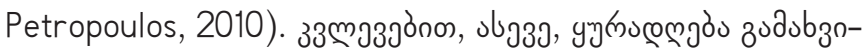

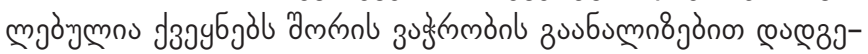

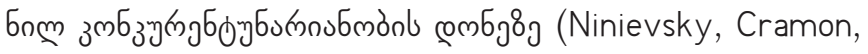
2009).

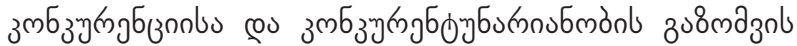

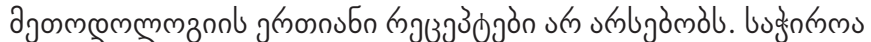

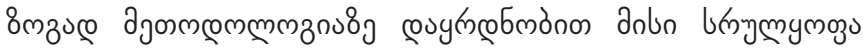

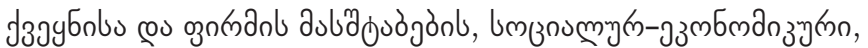

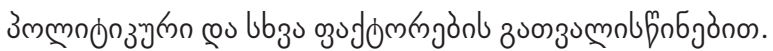

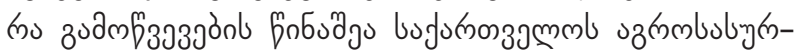

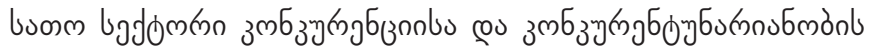

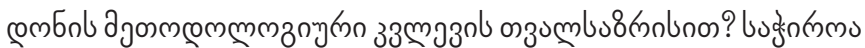

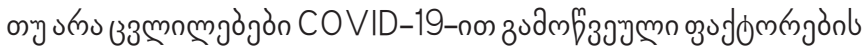

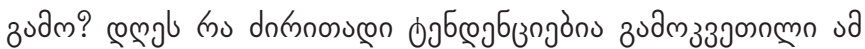
๓зumbu8कnonom?

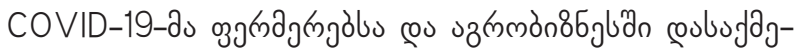

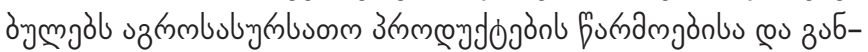

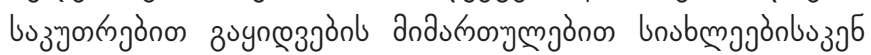

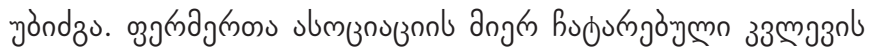

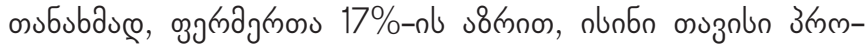

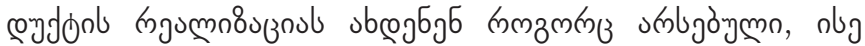

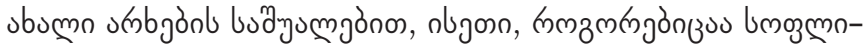

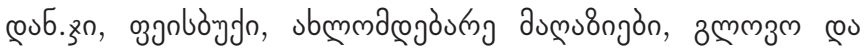

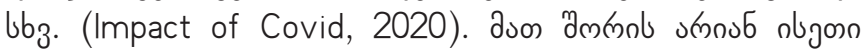

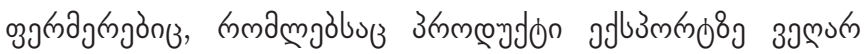

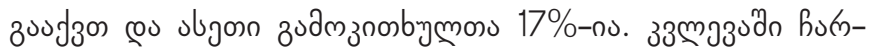

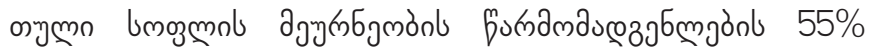

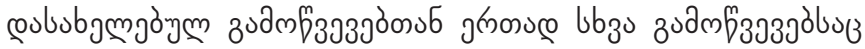

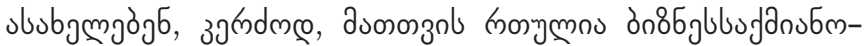

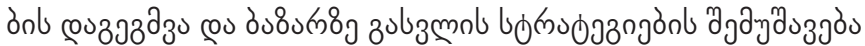

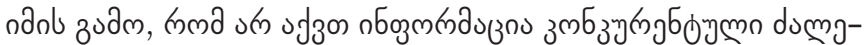

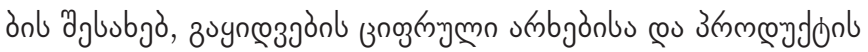

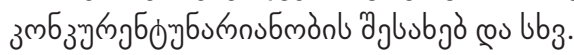

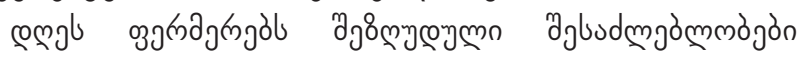

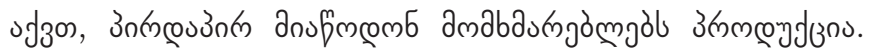

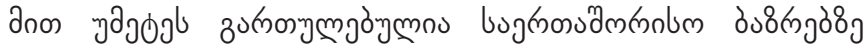

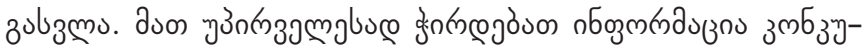

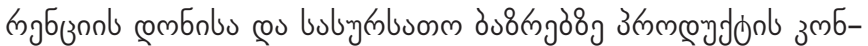

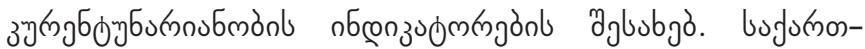




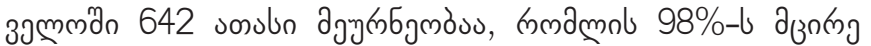

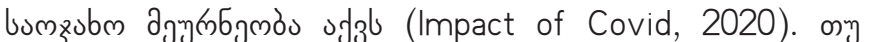

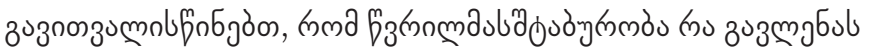

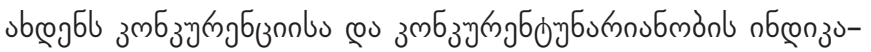

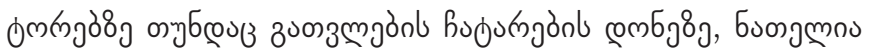

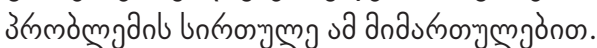

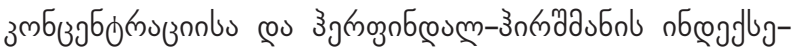

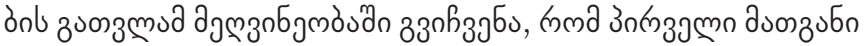

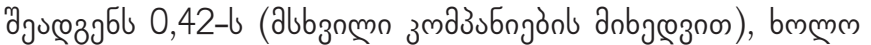

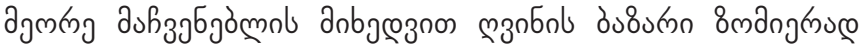

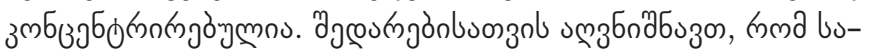

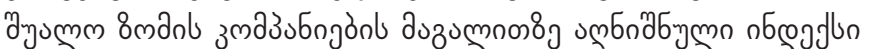

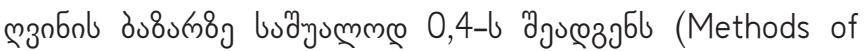
Market Analysis).

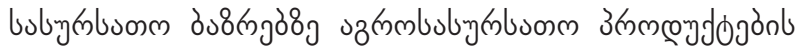

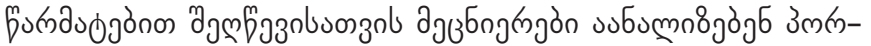

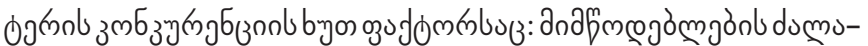

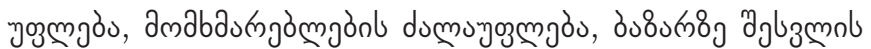

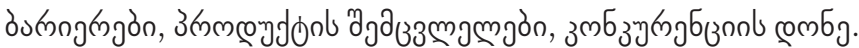

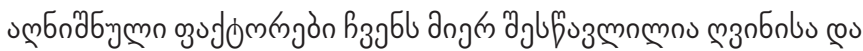

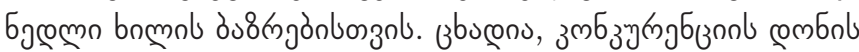
3u8maju nб

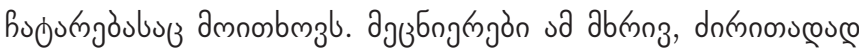

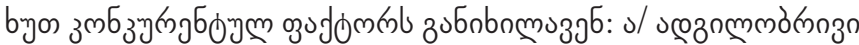

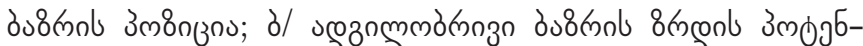

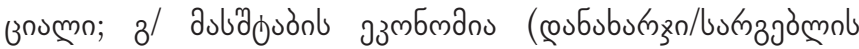

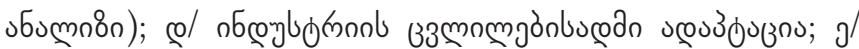
चzbmynon nб the Size, 2015).

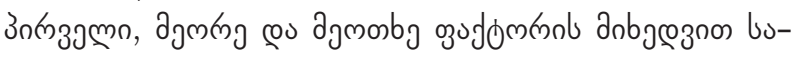

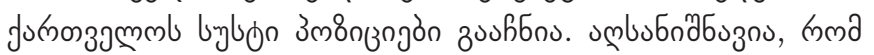

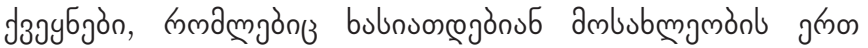

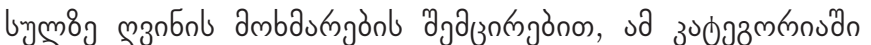

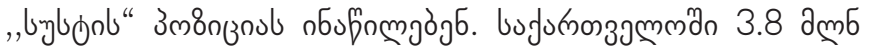

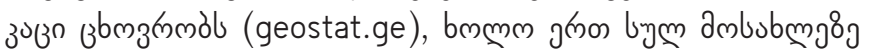

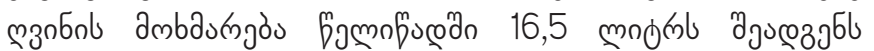
(Sterly, Jongeneel, Pabst, Silvis, Connor, Freshwater, Shobayashi, Kinoshita, Van Kooten, Zorn, 2018). duzubucoudg,

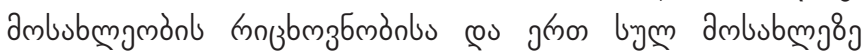

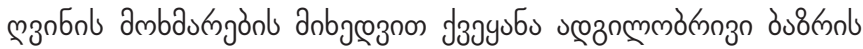

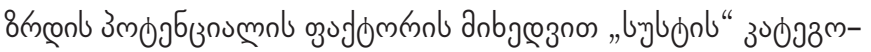

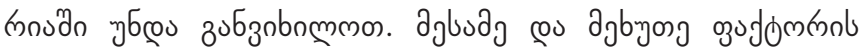

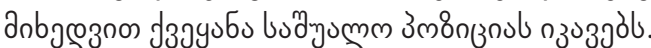

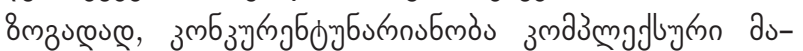

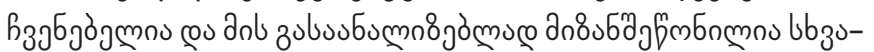

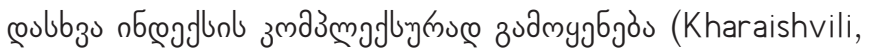

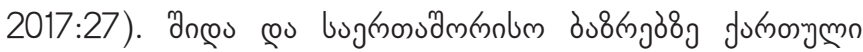

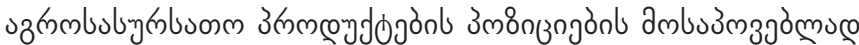

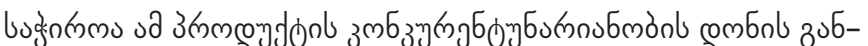
bu8ms mo.

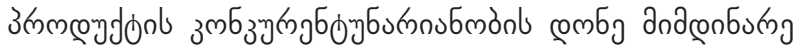

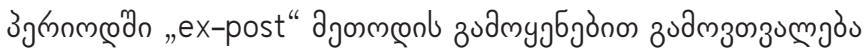

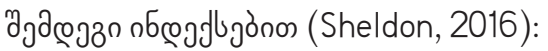

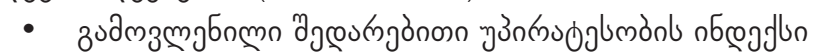
(RCA)

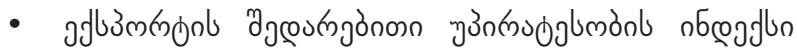

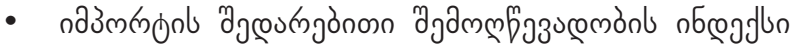
(RMP)

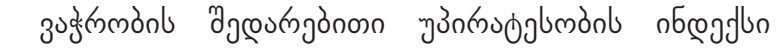

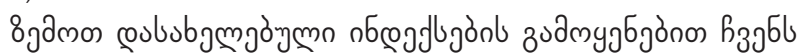

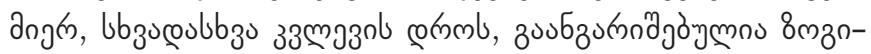

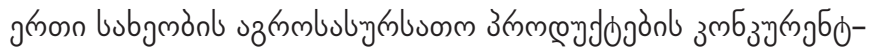

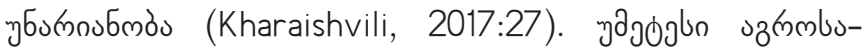

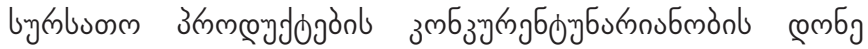

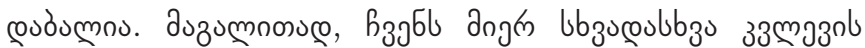

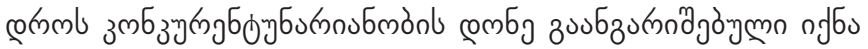

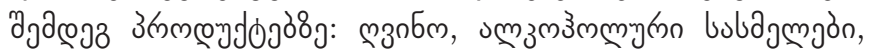

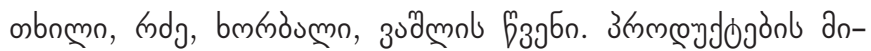

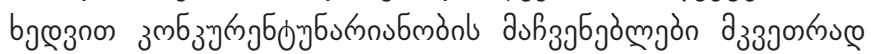

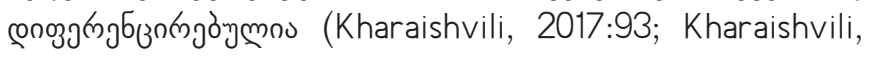

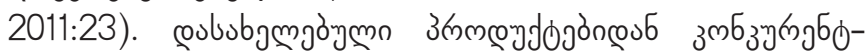
चб

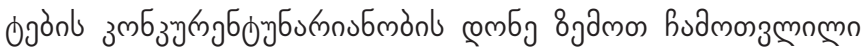

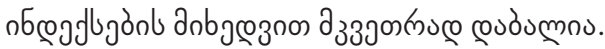

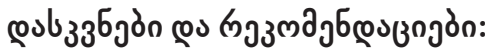

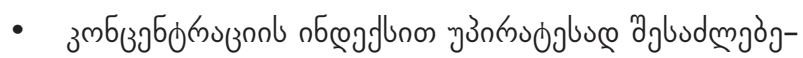

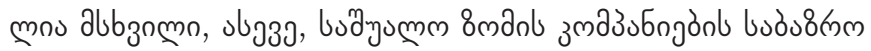

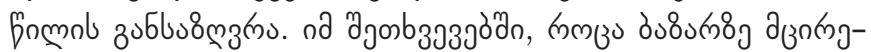

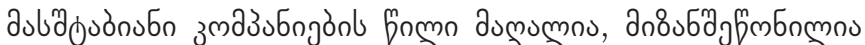

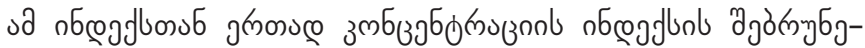

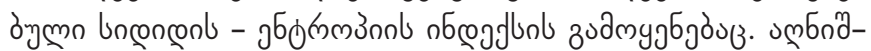

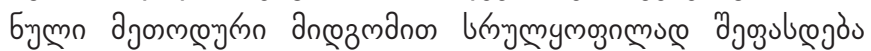

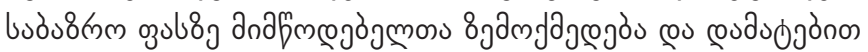
उubnbu8 fomons.

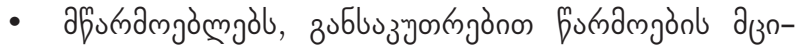

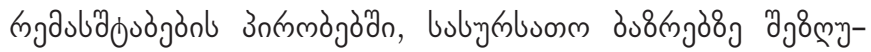

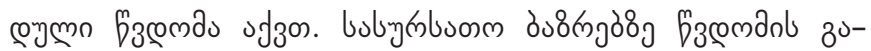

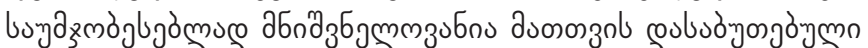

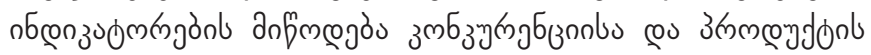

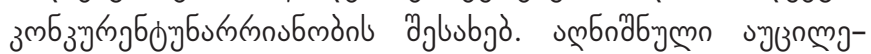
ajmn fnб

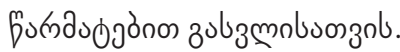

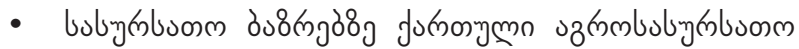

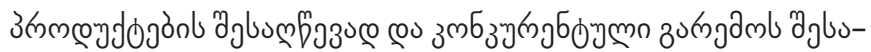

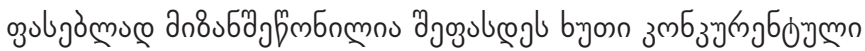




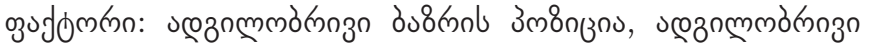

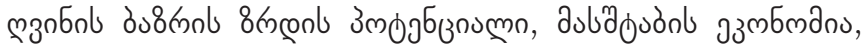

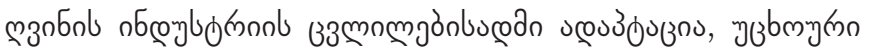

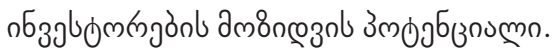

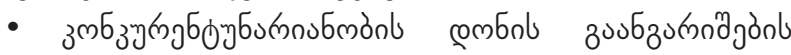

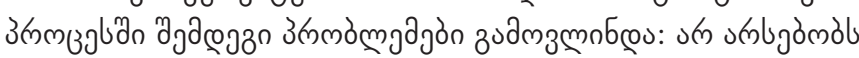

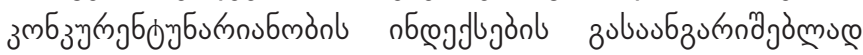

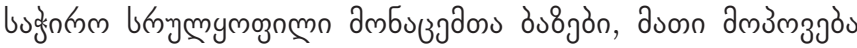

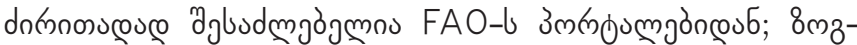

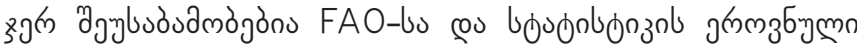

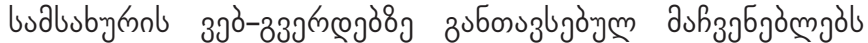

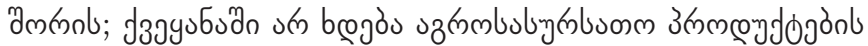

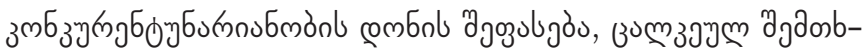

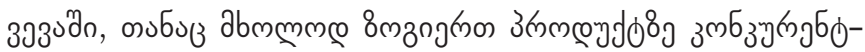

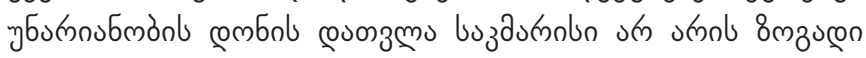

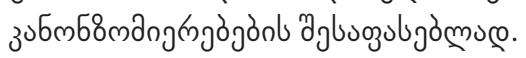

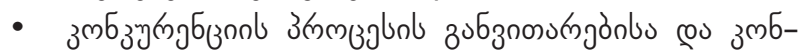

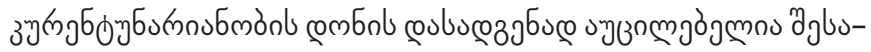

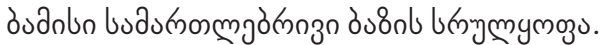

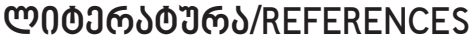

Methods of market analysis, http://competition.ge/ge/page2.php?p=4\&m=138 (In Georgian).

Gagnidze, I., (2012). Country Competitiveness and Clusters: History and Modernity, Tbilisi, 13. (In Georgian)

UN: Because of Coronavirus The World is at risk of starvation on a "biblical scale". (In Georgian)

https://1tv.ge/news/gaero-shi-ackhadeben-rom-koronavirusis-gamo-msoflio-bibliuri-masshtabis-shimshilis-riskis-winashea/ Donju, K., Don't a pandemic become a game of hunger. (In Georgian) https://bm.ge/ka/article/quotnu-davushvebt-romcovid19-gaxdes-shimshilis-tamashiquot---fao-s-gia-werili/52932

The EU economy is facing a historic recession. (In Georgian). Deutsche Welle:

https://feedc.com/post/54312

Erkomaishvili, G., (2016). Priority Directions of Economic Development Policy of Georgia, Tbilisi, 13. (In Georgian)

Impact of Covid 19 on Farmers and Agriculture, Farmers Association Study, 2020, (In Georgian)

https://gfa.org.ge/covid-19

Population and Demography, National Statistics Office of Georgia, (In Georgian).

http://geostat.ge/

Khaduri, N., Analysis of Competitiveness and Prospects of the Georgian Economy, Tbilisi, 2010, 4, (In Georgian). Kharaishvili,

E., Problems of Competition and Competitiveness in the Agro-Food Sector of Georgia, Tbilisi, 2011, 27, (In Georgian). Kharaishvili,

E., Problems of Competition and Competitiveness in the Agro-Food Sector of Georgia, Tbilisi, 2011, 91-93, (In

Georgian).

Kharaishvili, E., Wine Market and Competitive Models of Viticulture and Enology in Georgia, Tbilisi, 2017, 23, (In Georgian).

Agricultural competitiveness: The case of the European Union countries

https://doi.org/10.17221/133/2015-AGRICECON; Nowak, A., Kaminska, A. (2016): Agricultural competitiveness: The case of the European Union countries. Agric. Econ. - Czech, 62: 507-516.

Litvin, A., Coser, C., Competitiveness and Quality of Agrifood Products - Key Factors for the Success of Export Promotion, Scientific Papers Series Management, Economic Engineering in Agriculture and Rural Development 14, 3, 2014 PRINT ISSN 2284-7995, E-ISSN 2285-3952 http://managementjournal.usamv.ro/pdf/vol_14/art23.pdf

Analysis of the size and distribution of the impacts of agricultural trade at the firm and industry levels in developing countries, Food and Agriculture Organization of the United Nations Rome, 2015. http://www.fao.org/3/a-i5130e.pdf

Collins, 2004; Richard M., Castaldi, Susan Cholette, Mahmood Hussain, 2006.

Maslova V. , Zaruk N., Fuchs C., Avdeev M., (2019). Competitiveness of Agricultural Products in the Eurasian Economic Union. file:///C:/Users/Admin/Downloads/agriculture-09-00061.pdf

Measuring competitiveness in the EU market: a comparison between food industry and agriculture. Carraresi, L., and Banterle. A., 2008.

Nivievskyi, O., \& Cramon-Taubadel, S., Measuring and comparing the competitiveness of heterogeneous production systems in agriculture, 2009.

https://www.researchgate.net/publication/233562299_Measuring_and_comparing_the_competitiveness_of_ heterogeneous_production_systems_in_agriculture

Petropoulos, D., Kyriazopoulos, G., \& Papageorgiou, A., The Competitiveness of Agricultural Products (trade relations between Greece - Bulgaria), 2012

https://www.researchgate.net/publication/297577164_THE_COMPETITIVENESS_OF_AGRICULTURAL_PRODUCTS_trade_ relations_between_Greece_-_Bulgaria [accessed Mar 09 2020].

Papageorgiou, A., \& Petropoulos, D. (2010). The competitiveness of agricultural products in domestic and international market https://www.researchgate.net/publication/233562299_Measuring_and_comparing_the_competitiveness_of_ heterogeneous_production_systems_in_agriculture_ 
Research for AGRI Committee - A comparative analysis of global agricultural policies: lessons for the future CAP, 2018. https:// www.europarl.europa.eu/RegData/etudes/STUD/2018/629183/IPOL_STU\%282018\%29629183_EN.pdf

Sterly, S., Jongeneel, R., Pabst, H., Silvis, H., with contributions from Connor, J., (Australia), Freshwater, D., (US), Shohobayashi, M., (Japan), Kinoshita, Y., (Japan), Van Kooten, C., (Canada), and Zorn, A., (Switzerland). Research for AGRI Committee - A comparative analysis of global agricultural policies: lessons for the future CAP, 2018. https://www.europarl.europa.eu/ RegData/etudes/STUD/2018/629183/IPOL_STU\%282018\%29629183_EN.pdf

Sheldon, I. M. (2016). The competitiveness of agricultural product and input markets: A review and synthesis of recent research. Journal of Agricultural \& Applied Economics, 49(1):1-44 https://www.researchgate.net/publication/311972543_The_ competitiveness_of_agricultural_product_and_input_markets_A_review_and_synthesis_of_recent_research

Wine Institute, World Statistics, http://www.wineinstitute.org/resources/statistics

Weindlmaier, H. (1999): The competitiveness of the German food industry: methodological starting points for measurement and empirical results. Presentation. Fortieth annual meeting of the Society for Economic and Social Sciences in Agriculture 4. 